\title{
Negligible effect of eNOS palmitoylation on fatty acid regulation of contraction in ventricular myocytes from healthy and hypertensive rats.
}

Dol:

10.1007/s00424-017-1979-x

\section{Document Version}

Accepted author manuscript

Link to publication record in Manchester Research Explorer

Citation for published version (APA):

Zhang, Y., Jin, C. L., Wu, Y. N., Jang, J. H., Hao Zhao, Z., Oh, G. T., \& Kim, S. J. (2017). Negligible effect of eNOS palmitoylation on fatty acid regulation of contraction in ventricular myocytes from healthy and hypertensive rats. Pflügers Archiv European Journal of Physiology. https://doi.org/10.1007/s00424-017-1979-x

Published in:

Pflügers Archiv European Journal of Physiology

\section{Citing this paper}

Please note that where the full-text provided on Manchester Research Explorer is the Author Accepted Manuscript or Proof version this may differ from the final Published version. If citing, it is advised that you check and use the publisher's definitive version.

\section{General rights}

Copyright and moral rights for the publications made accessible in the Research Explorer are retained by the authors and/or other copyright owners and it is a condition of accessing publications that users recognise and abide by the legal requirements associated with these rights.

\section{Takedown policy}

If you believe that this document breaches copyright please refer to the University of Manchester's Takedown Procedures [http://man.ac.uk/04Y6Bo] or contact uml.scholarlycommunications@manchester.ac.uk providing relevant details, so we can investigate your claim.

\section{OPEN ACCESS}




\section{Negligible effect of eNOS palmitoylation on fatty acid regulation of contraction}

in ventricular myocytes from healthy and hypertensive rats

Chun Li Jin ${ }^{1,2}$, Yu Na Wu${ }^{1}$, Ji Hyun Jang ${ }^{1}$, Zai Hao Zhao ${ }^{1}$, Goo Taeg $\mathrm{Oh}^{4}$; Sung Joon Kim¹, Yin Hua Zhang ${ }^{1,2,3 *}$

${ }^{1}$ Department of Physiology \& Biomedical Sciences, Ischemic/hypoxic disease institute, Seoul National University, College of Medicine, Seoul, Korea; ${ }^{2}$ Yan Bian University Hospital, Yanji, Ji Lin, China; ${ }^{3}$ Division of Cardiovascular Sciences, University of Manchester, Manchester, UK; ${ }^{4}$ Department of Life Sciences, Ewha Womans University, Seoul, Korea

*Corresponding author: Yin Hua Zhang MD PhD

Department of Physiology \& Biomedical Sciences, Seoul National University, College of Medicine, Jongno Gu, 103 Dae Hak Ro, Seoul, Korea, 110-799;

E-mail: yinzhang87@gmail.com

Phone: +8227408223

Fax: +82 27639667 


\begin{abstract}
$S$-palmitoylation is an important post-translational modification that affects the translocation and the activity of target proteins in a variety of cell types including cardiomyocytes. Since endothelial nitric oxide synthase (eNOS) is known to be palmitoylated and the activity of eNOS is essential in fatty acid-dependent $\beta$-oxidation in muscle, we aimed to test whether palmitoylation of eNOS is involved in palmitic acid (PA)-regulation of left ventricular (LV) myocyte contraction from healthy (sham) and hypertensive (HTN) rats. Our results showed that PA, a predominant metabolic substrate for cardiac $\beta$ oxidation, significantly increased contraction and oxygen consumption rate (OCR) in LV myocytes from sham. L-NAME (N $\omega$-nitro-L-arginine methyl ester hydrochloride) or eNOS gene deletion prevented PAregulation of the myocyte contraction or OCR, indicating the pivotal role of eNOS in mediating the effects of PA in cardiac myocytes. PA increased the palmitoylation of eNOS in LV myocytes and depalmitoylation with 2 bromopalmitate $(2 \mathrm{BP}, 100 \mu \mathrm{M})$ abolished the increment. Furthermore, although PA did not increase eNOS-Ser ${ }^{1177}, 2 \mathrm{BP}$ reduced eNOS-Ser ${ }^{1177}$ with and without PA. Intriguingly, PA-induced increases in contraction and OCR were unaffected by $2 \mathrm{BP}$ treatment. In HTN, PA did not affect eNOS palmitoylation, eNOS-Ser ${ }^{1177}$ or myocyte contraction. However, 2BP diminished eNOS palmitoylation and eNOS-Ser ${ }^{177}$ in the presence and absence of PA but did not change myocyte contraction. Collectively, our results confirm eNOS palmitoylation in LV myocytes from sham and HTN rats and its up-regulation by PA in sham. However, such post-transcriptional modification plays negligible role in PA-regulation of myocyte contraction and mitochondrial activity in sham and HTN.
\end{abstract}

Keyword: cardiac myocyte, contraction, eNOS, oxygen consumption, palmitic acid, $S$-palmitoylation 


\section{Introduction}

Fatty acids are the predominant metabolic substrates that play important roles in cardiac metabolism and myocardial function [3,11]. Palmitic acid (PA, a 16-carbon saturated fatty acid) which is one of the most common saturated fatty acids for myocardial ATP, has been implicated in the regulation of cardiac contraction. However, the mechanism of this regulation is not fully understood. Recent evidence indicates that nitric oxide (NO) that is derived from endothelial NOS (eNOS) may play a critical role in the regulation of energy production and fatty acid utilization by muscle. For example, Le Gouill et al. demonstrated that oxygen consumption in muscle from eNOS gene knockout $\left(\mathrm{eNOS}^{--}\right)$mice is impaired compared to that in normal mice [8]. Furthermore, mitochondrial $\beta$-oxidation was reduced in $\mathrm{eNOS}^{-/-}$mice [8]. Since NO is well established to affect myocardial function, including the modulation of contraction, energetics (metabolism) and signaling pathways [5,12], eNOS may regulate PA-dependent myocyte contraction in the heart.

$S$-palmitoylation is an important post-translational modification that affects the translocation and the activity of target proteins in the cells and the reversible nature of palmitoylation provides a potential mechanism for protein shuttling between intracellular compartments [9]. eNOS is known to be palmitoylated [10] and it is possible that the activity of eNOS is altered to play a part in fatty acid $\beta$-oxidation in cardiac myocyte and regulate myocardial contractility. Recently, we have shown that eNOS protein expression is reduced in angiotensin II (Ang II) - induced hypertensive myocardium [7]. Reduced NO bioavailability is able to impair cardiac metabolism and reduce the capacity of fatty acid oxidation $[\mathbf{5 , 1 1}]$. We hypothesize that PA-regulation of myocyte contraction is impaired due to the lack of eNOS palmitoylation in hypertensive heart.

Here, we aimed to identify whether eNOS is palmitoylated by PA in left ventricular (LV) myocytes and how this post-translational modification affects eNOS activity (eNOS phosphorylation) in healthy heart and in hypertension. Furthermore, we aimed to identify whether eNOS palmitoylation contributes to PA-regulation of myocyte contraction and mitochondrial activity in healthy heart and whether this effect is diminished in hypertension. 


\section{Materials \& Methods}

Animals

Sprague-Dawley rats (10-12 weeks old, male) were used in the study. Hypertension was induced by infusing Ang II subcutaneously in rats using osmotic minipumps and they were paired with shamoperated group. Briefly, rats (of $8 \mathrm{wk}$ old, male) were anesthetized with isoflurane $(2.5 \%)$. An osmotic minipump (Alzet model 2004, DURECT Corporation, San Francisco, California, USA.) containing Ang II ( $200 \mu \mathrm{l}, 6 \mathrm{mM}$, infusion rate $125 \mathrm{ng} / \mathrm{min} / \mathrm{kg})$ was implanted in the mid-scapular region for 4 weeks. Sham-operated animals underwent the same surgical procedure, except for no pump insertion. Blood pressures were measured every 3 days for 4 weeks by Non-Invasive Blood Pressure System, tail-cuff method (CODA, Torrington, CT, USA).

eNOS knockout mice (21- to 25-wk-old males) were provided by Professor Goo Taeg Oh's Laboratory (Ewha Womans University, Seoul, Korea).

Isolation of left ventricular myocytes

LV myocytes were isolated enzymatically by Langendorff perfusion system as recently described [14]. Briefly, the rats were anesthetized with pentobarbital sodium $(30 \mathrm{mg} / \mathrm{kg}$, i.p. $)$ and the hearts were extracted and rapidly mounted onto the Langendorff perfusion system. The isolated heart was perfused with a nominally $\mathrm{Ca}^{2+}$-free solution for 10 minutes (in $\mathrm{mM}$ : $\mathrm{NaCl} 135, \mathrm{KCl} 5.4, \mathrm{MgCl}_{2} 3.5$, glucose 5, HEPES 5, $\mathrm{Na}_{2} \mathrm{HPO}_{4}$ 0.4, Taurine 20; pH titrated to 7.40 using $\mathrm{NaOH}$ ), followed by a further 8 minutes with the same solution with enzymes added (collagenase type 2, $1 \mathrm{mg} / \mathrm{ml}$, Worthington Biochemical Corporation, Lakewood, New Jersey, USA; protease, $0.1 \mathrm{mg} / \mathrm{ml}$, bovine serum albumin (BSA) $1.67 \mathrm{mg} / \mathrm{ml} ; \mathrm{Ca}^{2+} 0.05 \mathrm{mM}$ ). The $\mathrm{LV}$ free wall was isolated and placed in a separate flask containing fresh collagenase-only solution for 8 minutes (oxygenated and maintained at $37{ }^{\circ} \mathrm{C}$ ). Myocytes were harvested following a further 10 minute digestion period, washed and re-suspended in storage solution (in $\mathrm{mM}$ : $\mathrm{NaCl} 120, \mathrm{KCl} 5.4, \mathrm{MgSO}_{4}$ 5, $\mathrm{CaCl}_{2}$ 0.2, Napyruvate 5, glucose 5.5, Taurine 20, HEPES 10, D-mannitol 29, pH titrated to 7.40 using $\mathrm{NaOH}$ ). The myocyte suspension was stored at room temperature and cells were used within 8 hours of isolation.

\section{$S$-palmitoylation of eNOS}


For blocking the free thiol, LV myocytes were diluted with blocking buffer (100 mM HEPES, 1 mM EDTA, $2.5 \%$ SDS, $1 \%$ MMTS, $\mathrm{pH} 7.5)$ and incubated at $40^{\circ} \mathrm{C}$ for 4 hrs. Following acetone precipitation, the pellet was rinsed five times with $70 \%$ cold acetone and added in binding buffer (100 mM HEPES, 1.0 mM EDTA, 1\% SDS, pH 7.5). When employed, an equal volume of freshly prepared $2 \mathrm{M} \mathrm{NH} \mathrm{NH}_{2} \mathrm{OH}$ (pH adjusted to 7.0 with $2 \mathrm{M} \mathrm{NaCl}$ ) was added followed by prewashed thiopropyl-Sepharose (Sigma-Aldrich, Seoul, Korea). Binding reactions were carried out on a rotator at room temperature for $2.5 \mathrm{hrs}$. Each of supernatant was saved as the "total input." Resins were washed at least 7 times with binding buffer. For immunoblot analysis, elution was performed using 2X Laemmli sample buffer supplemented with $100 \mathrm{mM}$ DTT and heated to $95^{\circ} \mathrm{C}$ for $5 \mathrm{~min}$. The obtained sample was separated via SDS-PAGE and immunoblotting with eNOS. S-palmitoylation of eNOS was compared in LV myocytes before and after palmitic acid treatment [4].

\section{Measurement of LV myocyte contraction}

Isolated LV myocytes were superfused with normal Tyrode solution containing (in mM: $\mathrm{NaCl} \mathrm{141.4,}$ $\mathrm{KCl} 4, \mathrm{NaH}_{2} \mathrm{PO}_{4}$ 0.33, $\mathrm{MgCl}_{2}$ 1, HEPES 10, Glucose 5.5, $\mathrm{CaCl}_{2}$ 1.8, mannitol 14.5; $\mathrm{pH}$ titrated to 7.40 using $\mathrm{NaOH}$ ) in the recording bath mounted on the stage of a high-resolution inverted microscope (Diaphot 200, Nikon, JP). Changes in sarcomere length were measured in LV myocytes by using a video-sarcomere detection system (IonOptix Corporation, Milton, Massachusetts, USA). Measurements from at least 10 steady state contractions were averaged for each intervention. All experiments were carried out at $36 \pm 1{ }^{\circ} \mathrm{C}$ and field-stimulated at $2 \mathrm{~Hz}$ [14].

\section{Measurement of oxygen consumption rate (OCR) from LV myocytes}

OCR was measured using a fluorescence-based oxygen sensor (NeoFox, Ocean Optics, Dunedin, Florida, USA) connected to a phase measurement system (Instech Laboratories, Inc., Plymouth Meeting, Philadelphia, USA). The sensor was calibrated regularly (every week) according to the manufacturer's instructions. Briefly, isolated LV myocytes (density: $2 \times 10^{4} / \mathrm{ml}$ ) were suspended in normal Tyrode's solution and was placed in a sealed chamber $(300 \mu \mathrm{L})$. Record oxygen level in the 
chamber over 30 min period $\left(37^{\circ} \mathrm{C}\right)$ followed by about 5 min recordings with carbonyl cyanide-4(trifluoromethoxy) phenylhydrazone (FCCP, $20 \mu \mathrm{M}$ ) to evaluate maximum OC of the myocytes in the chamber. Changes in the OCR with PA with and without nNOS inhibitor were calculated over 30 min-period in sham and HTN.

\section{Immunoblotting}

LV myocytes were lysed in lysis buffer contained $50 \mathrm{mM}$ Tris- $\mathrm{HCl}(\mathrm{pH}$ 7.4), $100 \mathrm{mM} \mathrm{NaCl}, 1 \%$ Triton X-100, $5 \mathrm{mM}$ EDTA with protease/phosphatase inhibitor cocktail (Roche Diagnostics, Mannhaim, Germany). Cell lysates were then centrifuged at $14,000 \mathrm{~g}$ for $30 \mathrm{~min}$ at $4{ }^{\circ} \mathrm{C}$, and the supernatant were acquired. The protein concentration was determined by the Bradford assay. The protein sampled were mixed with Laemmli sample buffer, resolved by $6 \%$ and $10 \%$ SDS-PAGE, and transferred to poly-vinylidene difluoride membranes in $25 \mathrm{mM}$ Tris, $192 \mathrm{mM}$ glycine, and $20 \%$ methanol. Membranes were blocked in 1X TBS containing $1 \%$ Tween-20 and $5 \%$ bovine serum albumin (blocking solution) for $1 \mathrm{hr}$ at room temperature with gentle rocking, and incubated overnight at $4{ }^{\circ} \mathrm{C}$ with anti-eNOS (BD Transduction Laboratories), eNOS-Ser ${ }^{177}$ (BD Transduction Laboratories, San Francisco, California, USA), GAPDH (Santa Cruz, Dallas, Texas, USA) primary antibodies followed by relevant secondary antibodies after washing. Blots were developed by ECL plus Western blotting detection reagents (Amersham Bioscience, Piscataway, NJ, USA). Membranes were stripped using Pierce restore western blot stripping buffer (Thermo Scientific, Waltham, MA, USA) for $30 \mathrm{~min}$, and the relative densities were calculated after normalizing the intensity of each sample band to that of GAPDH.

\section{NO production}

NO production was detected indirectly by measuring nitrite content in LV myocytes using NO assay kit (Griess Reagent System, Sigma-Aldrich, Seoul, Korea). Briefly, LV myocytes were homogenized in HEPES Buffer solution (in mM: $\mathrm{NaCl} 141.4, \mathrm{KCl} 4, \mathrm{NaH}_{2} \mathrm{PO}_{4} \mathrm{0.33}, \mathrm{MgCl}_{2} 1$, HEPES 10, Glucose 5.5, $\mathrm{CaCl}_{2}$ 1.8, mannitol 14.5, mixtures of phosphatase inhibitors and protease inhibitors, $\mathrm{pH}$ titrated to 7.40 using $\mathrm{NaOH}$ ). After assaying the protein content with 
Bradford protocol, $50 \mu \mathrm{l}$ of the supernatant of lysates were added to wells and mixed with $50 \mu \mathrm{l}$ of sulfanilamide solution and incubated for 5-10 min at room temperature in the dark. The same volume of naphthylethylenediamine dihydrochloride (NED, Sigma-Aldrich) solution added to the wells and incubated for another 5-10 min at room temperature, protected from light. Absorbance of the mixture was measured with a microplate plate reader (at $540 \mathrm{~nm}$, PowerWave $^{\mathrm{TM}}$ XS Microplate Spectrophotometer, BioTek Instruments, USA). Sodium nitrite (Sigma-Aldrich) was used as a standard.

\section{Chemicals}

Angiotensin II (Ang II, $6 \mathrm{mM}$ ) was injected to the osmotic mini-pumps. S-methyl-L-thiocitrulline acetate salt (SMTC, $100 \mathrm{nM}$, Sigma) and N $\omega$-nitro-L-arginine methyl ester hydrochloride (L-NAME, $1 \mathrm{mM}$, Sigma) were used to target nNOS and NOS activity. Sodium nitroferricyanide (III) dehydrate (SNP, $10 \mu \mathrm{M}$, Sigma) was an exogenous NO donor. Palmitic acid (PA, $100 \mu \mathrm{M}$, Sigma) was used to stimulate fatty acid oxidation. 2-bromopalmitic acid (2BP, $100 \mu \mathrm{M}$, Sigma) was an inhibitor of $S$ palmitoylation. Carbonyl cyanide-4-(trifluoromethoxy) phenylhydrazone (FCCP, $20 \mu \mathrm{M}$, Sigma) was an uncoupling agent induced maximal oxygen consumption rate. Sodium hydrosulfite or hyposulfite $\left(\mathrm{Na}_{2} \mathrm{~S}_{2} \mathrm{O}_{4}\right.$, Sigma-Aldrich) was used in calibration for $\mathrm{OCR}$ by minimizing $\mathrm{O}_{2}$ level in the solution.

\section{Statistics}

Data are reported as mean \pm SEM. $\mathrm{N}$ indicates the number of cells used. For all comparisons, cells were obtained from a minimum of three hearts per treatment group per protocol. Data were analyzed using student's paired and unpaired $t$ test. A value of $p<0.05$ was considered to be statistically significant. Data are reported as mean \pm SEM.

\section{Results}

eNOS mediates PA-regulation of myocyte contraction 
Fig.1A \& D showed that PA $(100 \mu \mathrm{M})$ significantly increased sarcomere shortening in LV myocytes from sham rats $(p<0.0001$, control $v s$. PA in shams, $\mathrm{n}=13)$. However, the positive inotropic effect of PA was absent in the presence of a non-specific inhibitor of NOS, N $\omega$-nitro-L-arginine methyl ester hydrochloride (L-NAME, $1 \mathrm{mM}, 30 \mathrm{~min}-1 \mathrm{hr}),(p=0.26$, control $v s$. PA in pre-treatment of L-NAME in shams, $\mathrm{n}=8 ; p<0.001$, PA $v s$. L-NAME+PA in sham, $\mathrm{n}=13$ and $\mathrm{n}=8$, Fig.1B \& D) and in LV myocytes from eNOS ${ }^{--}$mice ( $p=0.2927$, control vs. PA in eNOS ${ }^{-/}$, Fig.1C \& E).

We performed additional experiments to observe whether eNOS affects mitochondrial activity (oxygen consumption rate, OCR) in LV myocytes from sham rats with and without eNOS inhibition and from eNOS ${ }^{-/-}$mice. As shown in Fig.2A, PA significantly increased OCR in LV myocytes from sham at control but not with L-NAME ( $p=0.004$, control $v s$. PA in shams, $\mathrm{n}=10$ and $\mathrm{n}=9 ; p=0.4935$, L-NAME $v s$. L-NAME+PA, $\mathrm{n}=8$ each; $p=0.1951$, control $v s$. L-NAME in sham, $\mathrm{n}=10$ and $\mathrm{n}=8$; $p=0.008, \mathrm{PA} v s$. L-NAME+PA, $\mathrm{n}=9$ and $\mathrm{n}=8$ ). This is in contrast to the inhibition of neuronal NOS (nNOS) with SMTC (100nM, 30min 1hr), where PA failed to affect the OCR ( $p=0.0239$, SMTC $v s$. SMTC+PA in shams, $n=8$ and $n=10$, Fig.2A). In line with this result, PA failed to increase myocyte shortening following SMTC-pretreatment (data not shown). In addition, PA did not induce an increase in OCR in LV myocytes from eNOS ${ }^{-/}$mice, on the contrary, PA reduced OCR in eNOS ${ }^{-/-}(p=0.0329$, control vs. $\mathrm{PA}$ in $\mathrm{eNOS}^{-/}$mice, $\mathrm{n}=6$ and $\mathrm{n}=7$, Fig. $2 \mathrm{~B}$ ). These data indicate that eNOS is essential in mediating PA-regulation of myocyte contraction through stimulating mitochondrial activity.

\section{S-palmitoylation of eNOS and its regulation of eNOS-phosphorylation and OCR}

Fig. 3A showed that PA $(100 \mu \mathrm{M})$ increased eNOS palmitoylation in LV myocytes from sham rats ( $p=0.023$, control vs. PA in sham, $\mathrm{n}=6$, Fig. $3 \mathrm{~A}$ ). Pre-treatment of LV myocytes with a palmitate analog that is the most commonly used to inhibit palmitoylation in cells, 2-bromopalmitic acid (2BP, $100 \mu \mathrm{M}, 10-30 \mathrm{~min})$, abolished PA-induced eNOS palmitoylation $(p=0.005$, PA $v s . \mathrm{PA}+2 \mathrm{BP}$ in sham, $n=6)$.

Furthermore, we aimed to observe whether the palmitoylation of eNOS affects its activity by 
regulating eNOS-Ser ${ }^{1177}$. As shown in Fig. 3B, eNOS-Ser ${ }^{1177}$ was not affected by PA in LV myocytes from sham. However, 2BP significantly reduced eNOS-Ser ${ }^{1177}$ with and without PA $(p=0.235$, control vs. PA; $p=0.02677$, control vs. $2 \mathrm{BP} ; p=0.00949$, PA vs. $\mathrm{PA}+2 \mathrm{BP} ; p=0.01294,2 \mathrm{BP} v s . \mathrm{PA}+2 \mathrm{BP}$ in sham, n=6, Fig. 3B). Paradoxically, the NO production (nitrite level relative to control), which was reduced by PA $(84 \%$ of control, $p=0.045, n=6)$, significantly increased by $2 \mathrm{BP}$ in sham $(112 \%, p=0.152, \mathrm{Ctr} v s .2 \mathrm{BP}, \mathrm{n}=6 ; 139 \%, p=0.028, \mathrm{PA}$ vs. $\mathrm{PA}+2 \mathrm{BP}, \mathrm{n}=6)$. Furthermore, neither SMTC nor L-NAME prevented the effect of $2 \mathrm{BP}(147 \%, p=0.03$, SMTC $v s$. SMTC+PA+2BP, $\mathrm{n}=8 ; 149 \%, p=0.02$, L-NAME $v s$. L-NAME $+\mathrm{PA}+2 \mathrm{BP}, \mathrm{n}=8$ ). Therefore, the effect of $2 \mathrm{BP}$ on eNOS-Ser $^{1177}$ does not associate with myocyte NO level in sham.

To examine whether eNOS palmitoylation affects the function of PA-dependent responses in LV myocytes, both OCR and myocyte contraction were detected with 2BP pre-treatment. As shown in Fig. 3C, pre-treatment LV myocytes with 2BP failed to affect PA-potentiation of OCR in sham ( $p=0.004$, control vs. PA; $p<0.0001,2 \mathrm{BP} v s . \mathrm{PA}+2 \mathrm{BP} ; p=0.0752$, PA $v s . \mathrm{PA}+2 \mathrm{BP}$, Fig.3C). NOS inhibition with L-NAME prevented PA-dependent OCR increase in the presence of $2 \mathrm{BP}(p<0.0001$, $\mathrm{PA}+2 \mathrm{BP} v s . \mathrm{L}-\mathrm{NAME}+\mathrm{PA}+2 \mathrm{BP}$, Fig. 3C).

Furthermore, treatment of LV myocytes with 2BP did not affect myocyte contraction with PA in sham ( $p<0.0001$, control $v s$. PA, $p=0.0015$, PA $v s$. PA+2BP, n=13, Fig.4A \& D). Pre-treatment of 2BP did not prevent PA-dependent myocyte contraction $(p=0.005$, 2BP $v s .2 \mathrm{BP}+\mathrm{PA}, \mathrm{n}=8$, Fig.4B \& E). Similarly, 2BP did not affect myocyte contraction in the presence of L-NAME, i.e. PA did not increase myocyte contraction in the presence of L-NAME and 2BP $(p=0.406$, L-NAME+PA $v s$. LNAME+PA+2BP, n=8, Fig. 4C \& F). These results exclude the effect of eNOS palmitoylation on eNOS-mediated and PA-induced myocyte contraction in sham.

\section{Palmitoylation of eNOS in PA-regulation of LV myocytes contraction and OCR from HTN rats}

In HTN, PA did not increase eNOS palmitoylation ( $p=0.58$, control $v s$. PA, $\mathrm{n}=4$, Fig.5A). However, 2BP significantly reduced eNOS palmitoylation in the presence and absence of PA ( $p=0.04$, PA $v s$. 
PA+2BP, Fig.5A), suggesting that basal eNOS is palmitoylated and PA does not affect it further. Similar to that in sham, 2BP significantly reduced eNOS-Ser ${ }^{1177}$ in HTN with and without PA (with PA, $p=0.038$, PA vs. PA+2BP; without PA, $p=0.05$, control vs. $2 \mathrm{BP}, \mathrm{n}=3$, Fig.5B). In addition, the NO production was significantly increased by $2 \mathrm{BP}$ in the presence of SMTC and L-NAME $(140 \%, p=0.02$ SMTC $v s$. SMTC+PA+2BP, $\mathrm{n}=8 ; 180 \%, p=0.01, \mathrm{~L}-\mathrm{NAME} v s . \mathrm{L}-\mathrm{NAME}+\mathrm{PA}+2 \mathrm{BP}$, $\mathbf{n = 8 ) . ~ U n e x p e c t e d l y , ~ 2 B P ~ r e d u c e d ~ P A - i n d u c e d ~ O C R ~ i n ~ H T N ~}(p=0.0177$, control and PA, $\mathrm{n}=8$ and $\mathrm{n}=9$; $p=0.0198$, PA $v s . \mathrm{PA}+2 \mathrm{BP}, \mathrm{n}=9$ and $\mathrm{n}=10 ; p=0.2419,2 \mathrm{BP} v s . \mathrm{PA}+2 \mathrm{BP}, \mathrm{n}=8$ and $\mathrm{n}=10$, Fig.5C).

Finally, the effect of PA on myocyte contraction and the involvement of eNOS palmitoylation are examined. As shown in Fig.6, PA-dependent positive inotropic effect was absent in HTN $(\boldsymbol{p}=\mathbf{0 . 3 8 9 6}$, control vs. PA, n=12, Fig.6A \& D). This effect was unaffected by 2BP $(p=0.069,2 \mathrm{BP} v s . \mathrm{PA}+2 \mathrm{BP}$ in HTN, n=5, Fig.6B \& E). NOS inhibition with L-NAME significantly increased myocyte contraction with PA and 2BP did not affect such an effect (Fig. 6C \& F), indicating the lack of an effect of eNOS and its palmitoylation on myocyte contraction in HTN. These results show clear evidence that eNOS is palmitoylated in LV myocytes from HTN and does not change myocyte contraction with or without PA.

\section{Discussion}

In the present study we showed convincing evidence that a long chain fatty acid, PA, significantly increased LV myocyte contraction from healthy rat heart. This effect was prevented by pharmacological inhibition of eNOS with L-NAME or with eNOS gene deletion $\left(\mathrm{eNOS}^{-/}\right)$. Furthermore, PA increased mitochondrial activity (OCR) which was prevented by L-NAME or in $\mathrm{eNOS}^{-1-}$. In contrast, inhibition of nNOS with SMTC did not affect PA-induced myocyte contraction or mitochondrial activity. These results suggest that the effects of PA on myocyte contraction and mitochondrial activity are mainly mediated by eNOS but not by nNOS. Immunoblotting experiments showed direct evidence that PA increased the palmitoylation of eNOS which was abolished by a potent blocker of palmitoylation, 2BP. Interestingly, depalmitoylation of eNOS with 2BP was 
associated with a reduction of eNOS phosphorylation at $\mathrm{Ser}^{1177}$. However, 2BP, either pre-treatment or post-treatment, did not affect the inotropic effect of PA or PA regulation of mitochondrial activity, excluding the role of eNOS-palmitoylation as the mediating mechanism. In addition, eNOS was shown to be palmitoylated in LV myocytes from HTN rats. Depalmitoylation of eNOS with 2BP did not affect LV myocyte contraction in the presence and absence of PA, despite its inhibitory effect on eNOS Ser ${ }^{1177}$ in HTN. To our knowledge, this is the first study to exam the influence of eNOS and its palmitoylation in the regulation of LV myocyte contraction following supplementation of a favorable metabolic substrate of the myocardium in healthy and hypertensive hearts.

Fatty acids are the predominant metabolic substrates for myocardial ATP and therefore play essential roles in the contractility of the heart. During disease progression, glucose oxidation or glycolysis becomes the predominant sources of energy whereas both glucose and fatty acid metabolism are downregulated in the failing myocardium [13]. Understanding the mechanisms regulating cardiac contractility through fatty acid-dependent metabolism in healthy and diseased hearts are important in better defining the molecular targets underlying cardiac contraction in these conditions. Here, our results showed that PA increased myocyte contraction and mitochondrial activity (OCR). It is possible that PA exerts these effects through promoting $\beta$-oxidation in the mitochondria because our additional studies showed that PA increased ATP production from LV myocytes and both myocyte contraction and ATP level were normalized following the inhibition of carnitine palmitoyltransferase, a key limiting factor of $\beta$-oxidation [6]. The effect of PA on myocyte contraction was absent in HTN, indicating functional remodeling of cardiac metabolism in regulating contraction under pressure-overload.

On the other hand, PA is also the essential substrate for a post-translational modification, $S$ palmitoylation. As such, PA may affect myocyte contraction through changing the activities of the mediator proteins. Intriguingly, our results showed that eNOS plays a critical role in mediating PAregulation of myocyte contraction. Furthermore, eNOS palmitoylation is significantly increased by PA, which was reversed by pre-treatment of the inhibitor, 2BP. It is well established that the 
palmitoylation of eNOS is associated with its translocation to plasma membrane (caveolae) and increased its activity in endothelial cells [10]. There are evidences showed the interplays between eNOS palmitoylation and phosphorylation, that is, inhibition of eNOS palmitoylation with another potent inhibitor of palmitoylation, triacsin C, significantly reduced eNOS phosphorylation in cultured endothelial cells [1]. Paradoxically, triacsin C increased NO level in these experiments [1]. Similar to the responses in the report, our results showed that eNOS-Ser ${ }^{1177}$ was significantly reduced by $2 \mathrm{BP}$ in the presence and absence of PA. Moreover, in line with the results shown in endothelial cells [1], 2BP increased NO production in LV myocytes before and after PA supplementation. However, neither LNAME nor SMTC affected the increment of NO, excluding the roles of eNOS or nNOS. Although 2BP is a potent and reliable inhibitor of palmitoylation, it has been shown to exert off-target effects [2]. Therefore, 2BP may regulate NO level in myocyte samples through alternative mechanisms - it is speculative to suggest that 2BP increases cellular NO by preventing cysteine residues of NO-binding proteins. Nevertheless, 2BP did not affect OCR with or without PA supplementation, i.e. PA increased OCR in 2BP pre-treated LV myocytes, suggesting that 2BP does not affect the functional regulation of PA on the mitochondria in LV myocytes (despite increased NO level). Accordingly, 2BP was used to exam the effect of de-palmitoylation on PA-regulation of myocyte contractility. Similar to its effect on mitochondrial activity, 2BP did not affect myocyte contraction, either pretreated or post-treated, demonstrating that de-palmitoylation process, eNOS depalmitoylation in particular, is not the major mechanism mediating PA-regulation of myocyte contraction.

In HTN, cardiac NOSs are remodeled, exhibiting reduced eNOS protein level and greater nNOS protein expression and activity [7]. Furthermore, the residual eNOS was palmitoylated in HTN, judging from the significant reduction of eNOS palmitoylation by $2 \mathrm{BP}$ with and without PA. Palmitoylation of eNOS may have maintained the activity of eNOS since eNOS-Ser ${ }^{1177}$ was minimized by $2 \mathrm{BP}$ and $2 \mathrm{BP}$ reduced PA-dependent OCR increment in HTN. However, the significance of this modification remains uncertain. It is possible that reduced eNOS activity underlie the lack of PA-dependent inotropic response on LV myocytes in HTN, regardless of its palmitoylation 
since 2BP did not affect myocyte contraction in HTN. Inhibition of cardiac NOSs with L-NAME significantly increased myocyte contraction in HTN, similar results were shown with SMTC, suggesting nNOS modulation of myocyte contraction by PA in HTN. Notably, 2BP did not affect myocyte contraction even in the presence of L-NAME, excluding the involvement of eNOS palmitoylation in myocyte contraction by PA in HTN.

Taken together, our observations suggest a role of eNOS in mediating PA-regulation of myocyte contraction in healthy rat heart through stimulating mitochondrial activity. Although PA increases eNOS palmitoylation and maintains the phosphorylation of eNOS at $\mathrm{Ser}^{1177}$, we present clear evidence to show that eNOS palmitoylation is not the main mechanism to mediate PA-dependent inotropic response in healthy heart or the lack of this increment in HTN. The significance of eNOS and its post-transcriptional modification in regulating cardiac metabolism and contraction in healthy and diseased heart needs further investigation, which may provide novel insights into therapeutic targets to improve myocardial contractile function.

Conflicts of interests: none declared.

Sources of Funding

This research was supported by Basic Science Research Program through the National Research Foundation of Korea (NRF) funded by the Ministry of Education, Science and Technology (2013068067); by the Brain Korea 21 Graduate Programme of the Korean Ministry of Education, Science and Technology, Seoul National University Hospital, the Korean Society of Hypertension (2013), SK Telecom Research Fund (no. 3420130290) and from the National Natural Science Foundation of China (NSFC, 31460265).

\section{Reference}

1. Blakeman N, Weis M (2014). The interplay between eNOS palmitoylation and phosphorylation: the triacsin C effect. FASEB J 28 Supplement 1075. 9 (abstract). 
2. Davda D, El Azzouny MA, Tom CT, Hernandez JL, Majmudar JD, Kennedy RT, Martin BR (2013) Profiling targets of the irreversible palmitoylation inhibitor 2-bromopalmitate. ACS Chem Biol 8:1912-7 DOI 10.1021/cb400380s

3. Doenst T, Nguyen TD, Abel ED (2013) Cardiac metabolism in heart failure: implications beyond ATP production. Circ Res 113:709-24 DOI: 10.1161/CIRCRESAHA.113.300376.

4. Forrester MT, Hess DT, Thompson JW, Hultman R, Moseley MA, Stamler JS, Casey PJ (2011) Site-specific analysis of protein S-acylation by resin-assisted capture. J Lipid Res 52:393-8 DOI 10.1194/jlr.D011106

5. Hamilton DJ, Zhang A, Li S, Cao TN, Smith JA, Vedula I, Cordero-Reyes AM, Youker KA, Torre-Amione G, Gupte AA (2016) Combination of angiotensin II and l-NG-nitroarginine methyl ester exacerbates mitochondrial dysfunction and oxidative stress to cause heart failure. Am J Physiol Heart Circ Physiol 310:H667-80 DOI: 10.1152/ajpheart.00746.

6. He L, Kim T, Long Q, Liu J, Wang P, Zhou Y, Ding Y, Prasain J, Wood PA, Yang Q (2012). Carnitine palmitoyltransferase-1b deficiency aggravates pressure overload-induced cardiac hypertrophy caused by lipotoxicity. Circulation 126(14):1705-16. doi: 10.1161/CIRCULATIONAHA.111. 075978.

7. Jin CZ, Jang JH, Kim HJ, Wang Y, Hwang IC, Sadayappan S, Park BM, Kim SH, Jin ZH, Seo EY, Kim KH, Kim YJ, Kim SJ, Zhang YH (2013) Myofilament Ca2+ desensitization mediates positive lusitropic effect of neuronal nitric oxide synthase in left ventricular myocytes from murine hypertensive heart. J Mol Cell Cardiol 60:107-15 DIO: 10.1016/j.yjmcc.2013.04.017

8. Le Gouill E, Jimenez M, Binnert C, Jayet PY, Thalmann S, Nicod P, Scherrer U, Vollenweider P (2007) Endothelial nitric oxide synthase (eNOS) knockout mice have defective mitochondrial betaoxidation. Diabetes 56:2690-6 DOI: 10.2337/db06-1228

9. Linder ME, Deschenes RJ (2007) Palmitoylation: policing protein stability and traffic. Nat Rev Mol Cell Biol 8:74-84 DOI: 10.1038/nrm2084 
10. Liu J, Garcia-Cardena G, Sessa WC (1996) Palmitoylation of endothelial nitric oxide synthase is necessary for optimal stimulated release of nitric oxide: implications for caveolae localization. Biochemistry 35:13277-81 DOI 10.1021/bi961720e

11.Lopaschuk GD, Ussher JR, Folmes CD, Jaswal JS, Stanley WC (2010) Myocardial fatty acid metabolism in health and disease. Physiol Rev 90:207-58 DOI: 10.1152/physrev.00015.2009.

12. Massion PB, Feron O, Dessy C, Balligand JL (2003) Nitric oxide and cardiac function: ten years after, and continuing. Circ Res 93:388-98 DOI 10.1161/01.RES.0000088351.58510.21

13. Pellieux C, Montessuit C, Papageorgiou I, Lerch R (2009) Angiotensin II downregulates the fatty acid oxidation pathway in adult rat cardiomyocytes via release of tumour necrosis factor-alpha. Cardiovasc Res 82:341-50 DOI 10.1093/cvr/cvp004

14. Zhao ZH, Jin CL, Jang JH, Wu YN, Kim SJ, Jin HH, Cui L, Zhang YH (2016) Assessment of Myofilament Ca2+ Sensitivity Underlying Cardiac Excitation-contraction Coupling. J Vis Exp 10.3791/54057 DOI: $10.3791 / 54057$

\section{Figure legend}

Fig.1 eNOS is essential in mediating PA-regulation of myocyte contraction. Changes in sarcomere length were measured in LV myocytes by using a video-sarcomere detection system (IonOptix Corp). A\&B, D PA (Palmitic acid, $100 \mu \mathrm{M}$ ) significantly increased sarcomere shortening in LV myocytes from sham rats (sarcomere shortening, $p<0.0001, \mathrm{n}=13$ ). The positive inotropic effect of PA was prevented by L-NAME pre-treatment $(1 \mathrm{mM}, 30 \mathrm{~min}-1 \mathrm{hr}$ ) (sarcomere shortening, $p<0.001$, PA $v s . \mathrm{L}$ NAME+PA in sham, $\mathrm{n}=8$ ). $\mathbf{C} \& \mathbf{E}$ PA failed to affect myocyte contraction from $\mathrm{eNOS}^{-1-}$ mice. (sarcomere shortening, $p=0.2927, \mathrm{n}=7$ )

Fig.2 eNOS regulation of mitochondrial activity (oxygen consumption rate, OCR) in LV myocytes from rats and $\mathrm{eNOS}^{-/-}$mice. OCR was measured using a fluorescence-based oxygen sensor (NeoFox, 
Ocean Optics) connected to a phase measurement system (Instech). Bars represent average values \pm SEM. A PA significantly increased OCR in LV myocytes from sham at control. L-NAME (1mM, 30min 1hr) prevented PA-induced OCR increase, but nNOS with SMTC (100nM, 30min 1hr) failed to affect the OCR by PA (OCR, $\mu$ M.30min, $p<0.0001$, control $v s$. PA; $p=0.008$, PA $v s$. L-NAME+PA; $p=0.0239$, SMTC $v s$. SMTC+PA in shams). B PA did not induce an increase in OCR in eNOS ${ }^{--}$ mice (OCR, $\mu \mathrm{M} .30 \mathrm{~min}, p=0.0329$, control vs. PA). C Flow chart shows: eNOS mediates PAregulation of myocyte contraction through stimulating mitochondrial activity (oxygen consumption rate) in cardiac myocytes.

Fig.3 The palmitoylation and phosphorylation of eNOS by PA and its effects on mitochondrial activity in sham LV myocyte. A PA (Palmitic acid, $100 \mu \mathrm{M}$ ) increased eNOS-palmitoylation in LV myocytes from sham rats $(p=0.023$, control vs. PA) and 2BP $(100 \mu \mathrm{M})$ prevented eNOSpalmitoylation $(p=0.005$, PA $v s$. PA+2BP in sham, $\mathrm{n}=6)$. $\mathbf{B}$ eNOS-Ser ${ }^{1177}$ was not increased by PA in LV myocytes from sham ( $p=0.235$, control $v s$. PA). 2BP significantly reduced both basal eNOS$\operatorname{Ser}^{1177}$ and eNOS-Ser ${ }^{1177}$ with PA in sham $(p=0.02677$, control $v s .2 \mathrm{BP} ; p=0.00949$, PA $v s . \mathrm{PA}+2 \mathrm{BP}$; $p=0.01294,2 \mathrm{BP} v s . \mathrm{PA}+2 \mathrm{BP})$. C 2BP showed negligible effect on PA-regulation of OCR; NOS inhibition with L-NAME prevented PA-dependent OCR increase.

Fig.4 Effect of eNOS-palmitoylation on PA-induced myocyte contraction in sham. A, B, D\&E Post or pre-treatment of LV myocytes with $2 \mathrm{BP}$ did not reduce PA-induced myocyte contraction (sarcomere shortening, $p=0.0015$, PA vs. $\mathrm{PA}+2 \mathrm{BP}$, post-treatment $2 \mathrm{BP}, \mathrm{n}=13 ; p=0.005,2 \mathrm{BP} v s$. 2BP+PA, pre-treatment, $\mathrm{n}=8$ ). $\mathbf{C} \boldsymbol{E} \mathbf{F}$ NOS inhibition (L-NAME, 1mM, 30min -1hr) abolished PAdependent increase of myocyte contraction even in the presence of 2BP in sham (Sarcomere shortening, $p=0.406$, L-NAME+PA $v s$. L-NAME+PA+2BP, $\mathrm{n}=8$ ). Bars represent average values \pm SEM. 
Fig.5 Palmitoylation of eNOS in LV myocytes from hypertensive rats and the effects on OCR

A In hypertension, PA did not increase eNOS palmitoylation ( $p=0.58$, control $v s$. PA in HTN, $\mathrm{n}=4)$. However, 2BP significantly reduced basal eNOS palmitoylation in the presence and absence of PA $\left(p=0.04, \mathrm{PA} v s . \mathrm{PA}+2 \mathrm{BP}\right.$ in HTN). B Similar to that in sham, 2BP significantly reduced eNOS-Ser ${ }^{1177}$ in $\mathrm{HTN}$ with and without PA ( $p=0.038$, PA $v s . \mathrm{PA}+2 \mathrm{BP} ; p=0.05$, control $v s .2 \mathrm{BP}, \mathrm{n}=3)$. C $2 \mathrm{BP}$ reduced PA-induced OCR in HTN ( $p=0.0177$, control and PA, $\mathrm{n}=8$ and $\mathrm{n}=9 ; p=0.0198$, PA $v s$. $\mathrm{PA}+2 \mathrm{BP}, \mathrm{n}=9$ and $\mathrm{n}=10 ; p=0.2419,2 \mathrm{BP} v s . \mathrm{PA}+2 \mathrm{BP}$ in $\mathrm{HTN}, \mathrm{n}=8$ and $\mathrm{n}=10)$.

Fig.6 Effect of 2BP on LV myocyte contraction from hypertensive rats. A\&D PA-dependent positive inotropic effect was absent in HTN ( $p=0.3896$, control $v s$. PA in HTN, $\mathrm{n}=12$ ). B\&E PA-dependent positive inotropic effect was unaffected by $2 \mathrm{BP}$ pre-treatment $(p=0.069,2 \mathrm{BP} v s$. $\mathrm{PA}+2 \mathrm{BP}$ in HTN, $\mathrm{n}=5)$. $\mathbf{C} \& \mathbf{F}$ NOS inhibition with L-NAME significantly increased myocyte contraction with PA $(p=0.001, \mathrm{~L}-\mathrm{NAME} v s . \mathrm{L}-\mathrm{NAME}+\mathrm{PA}$ in HTN, $\mathrm{n}=7), 2 \mathrm{BP}$ did not affect such an effect $(p=0.2668, \mathrm{~L}-$ $\mathrm{NAME}+\mathrm{PA} v s . \mathrm{L}-\mathrm{NAME}+\mathrm{PA}+2 \mathrm{BP}$ in $\mathrm{HTN}, \mathrm{n}=7$ ). 


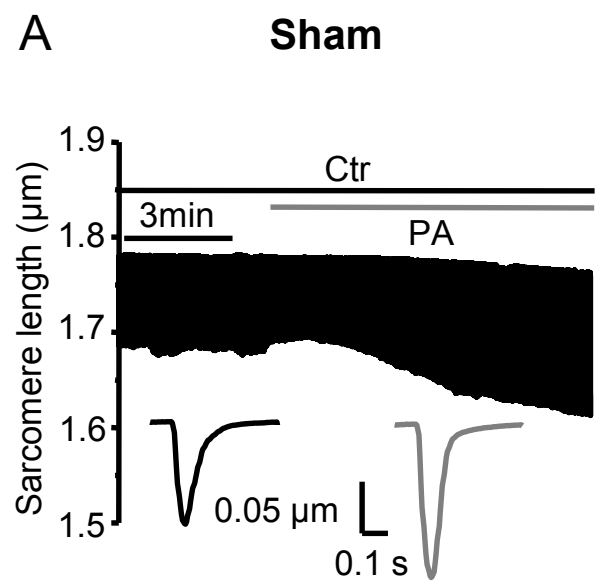

B

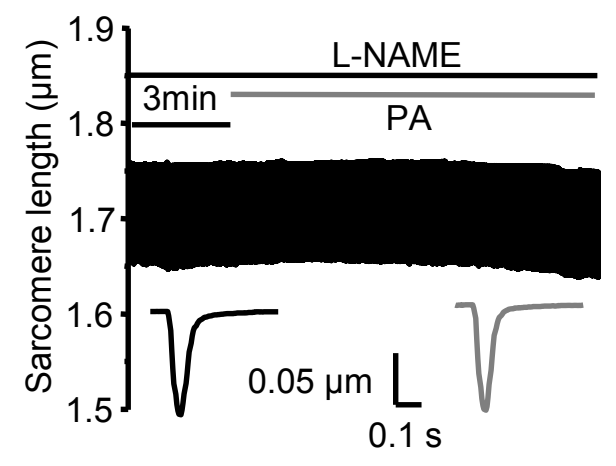

C eNOS KO

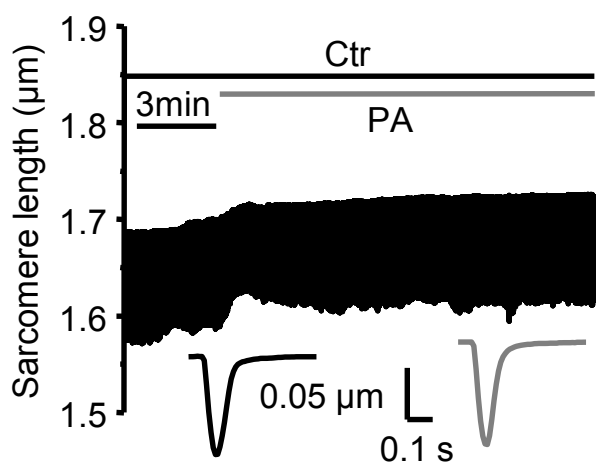

E eNOS KO

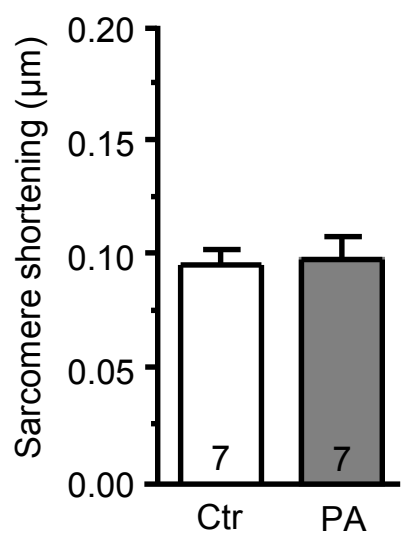


A

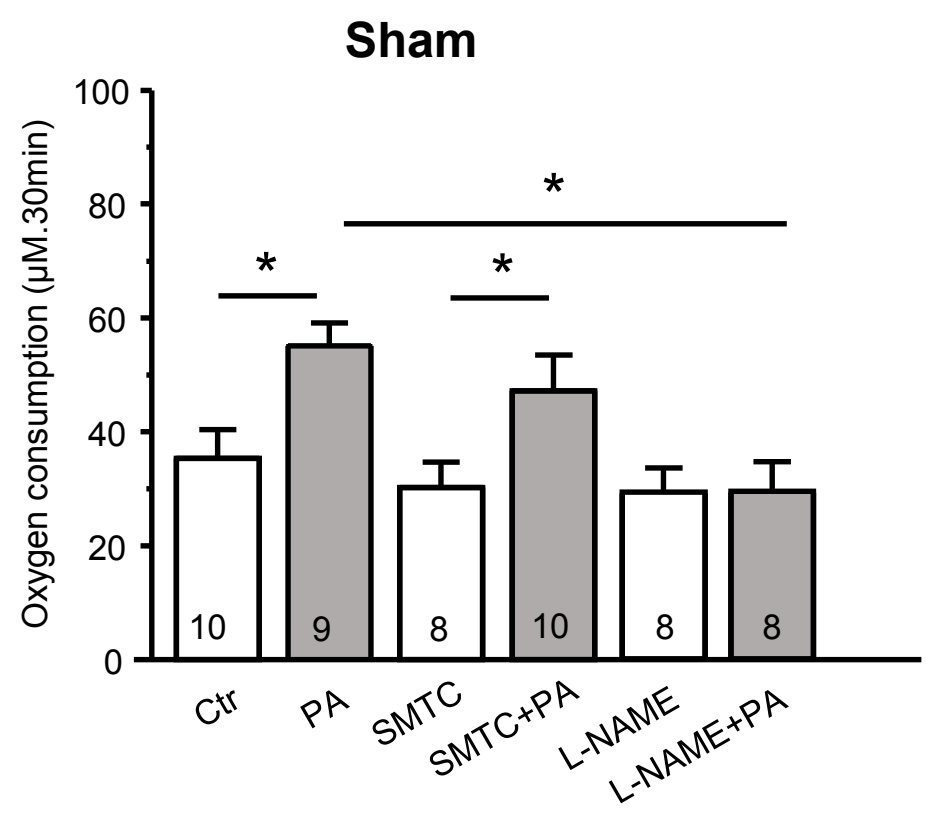

B

eNOS KO

C

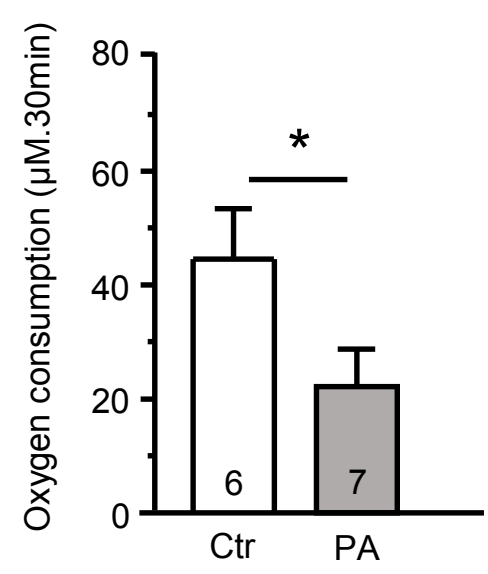

Palmitic acid $\downarrow$ eNOS Mitochondrial activity

Contraction 
A

Sham
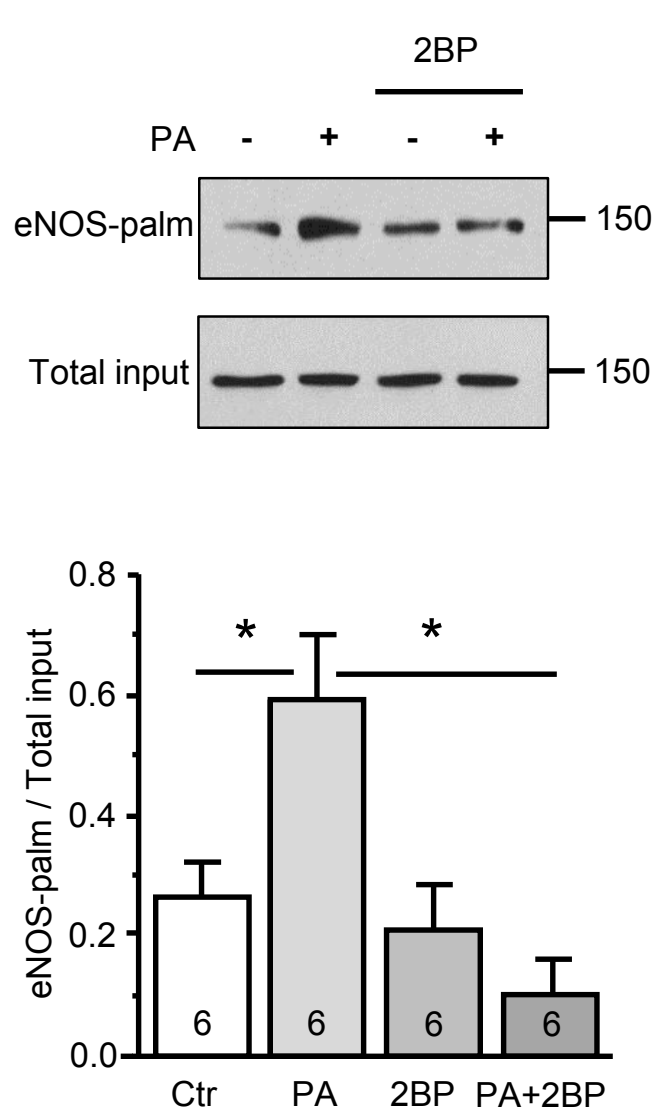

B

Sham
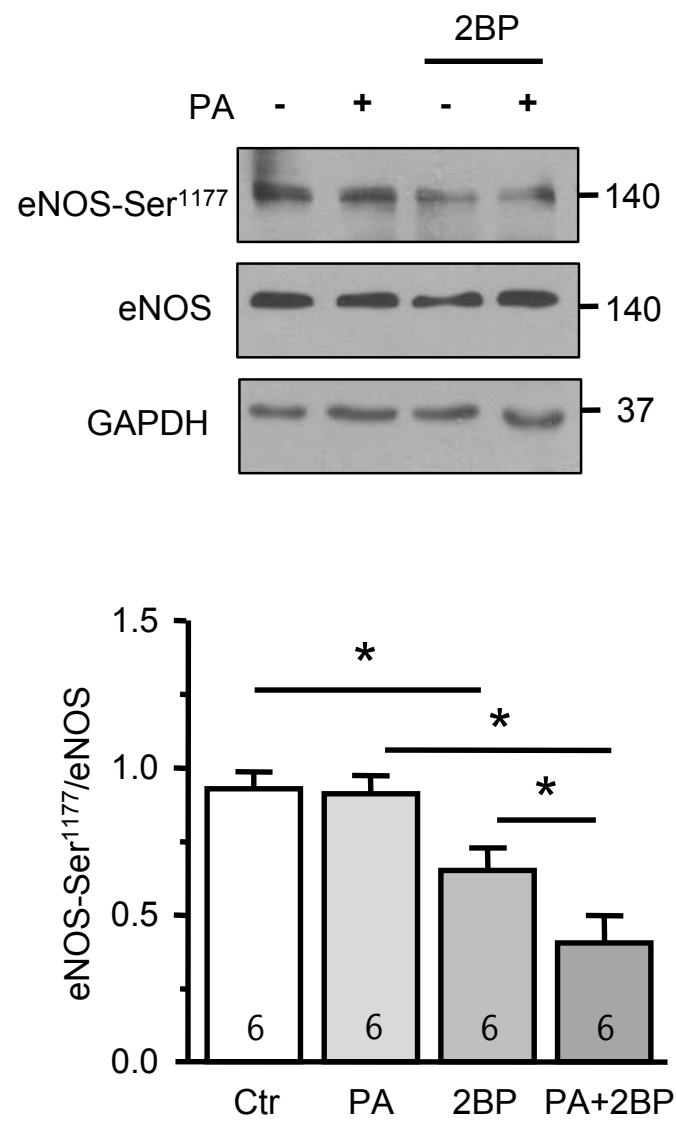

C

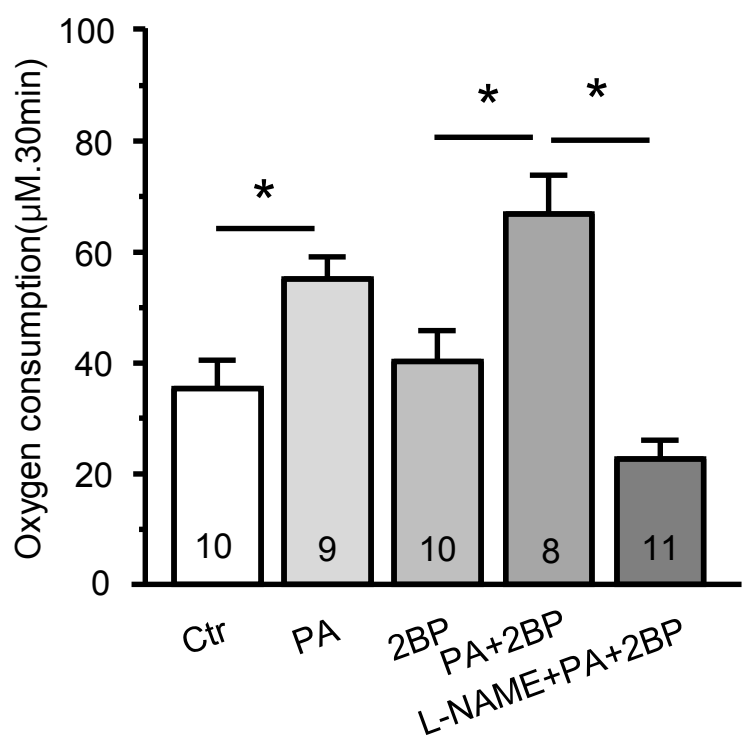

Sham 


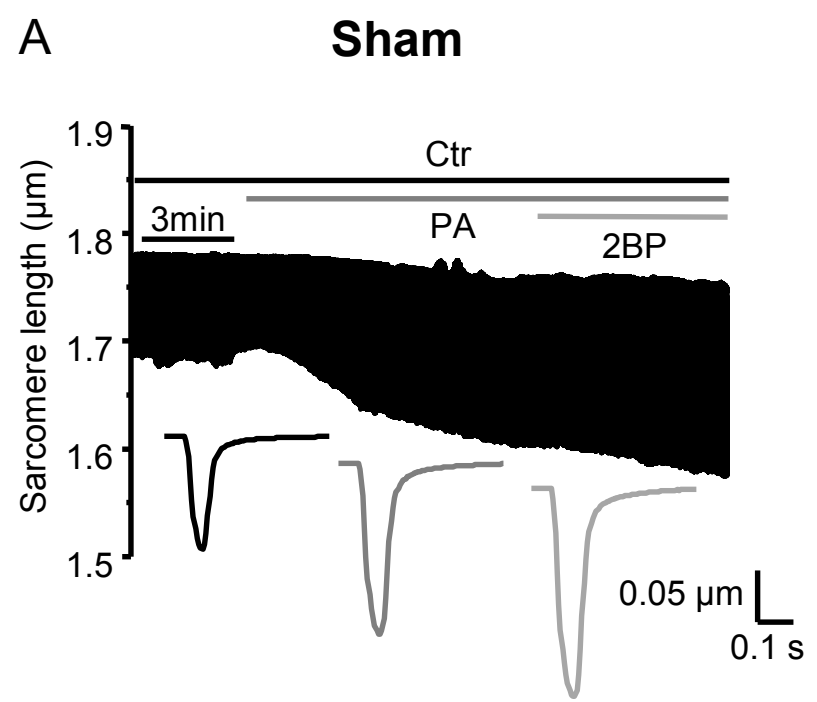

B

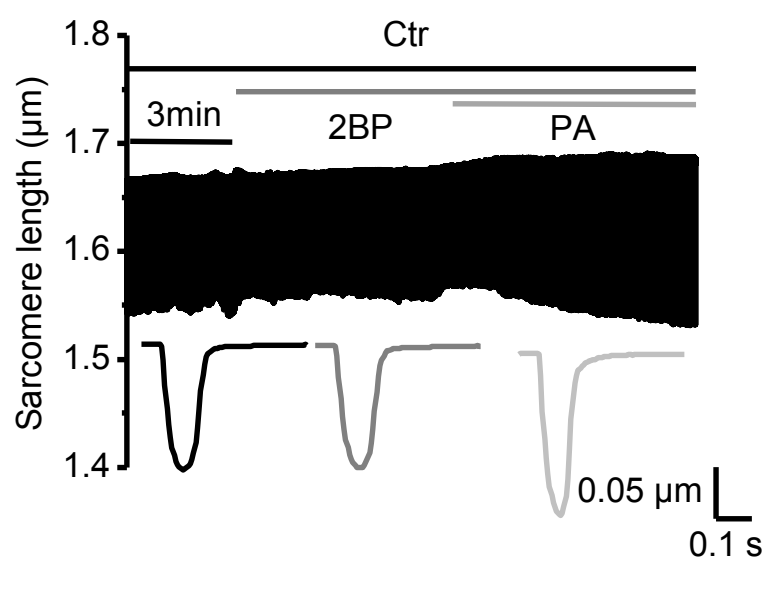

D

Sham

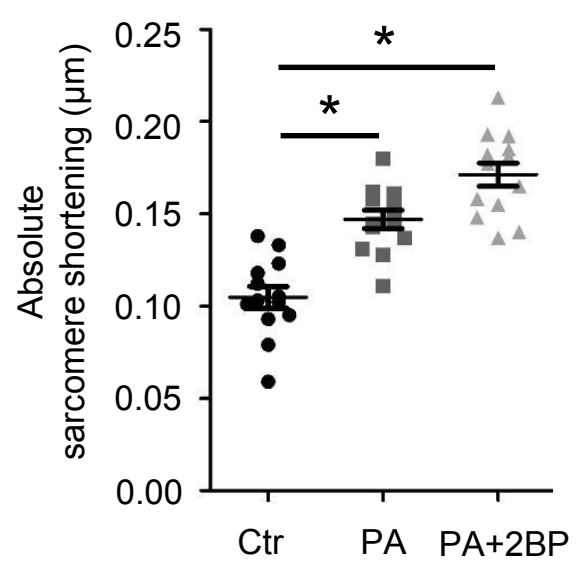

E

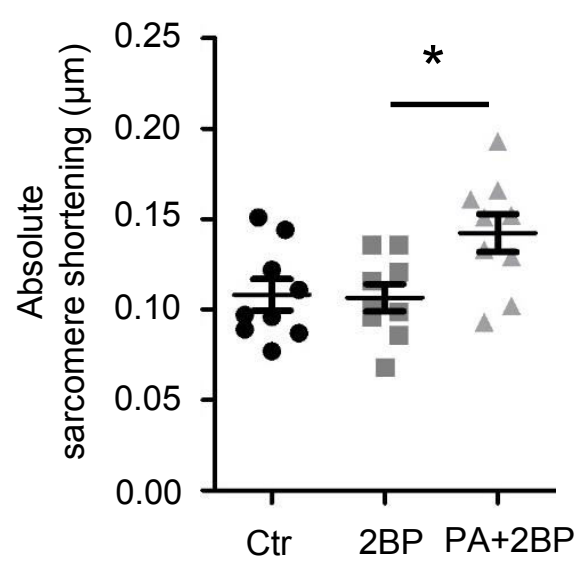

C Sham

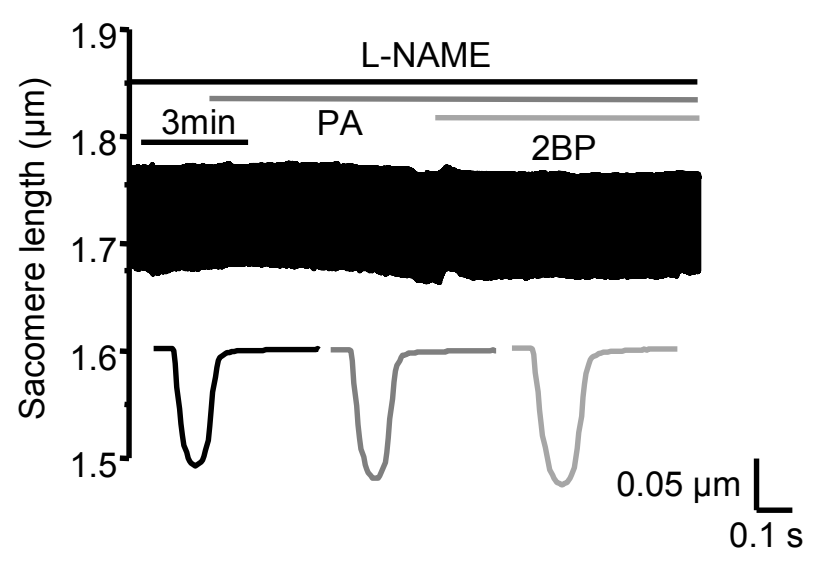

$\mathrm{F}$

Sham

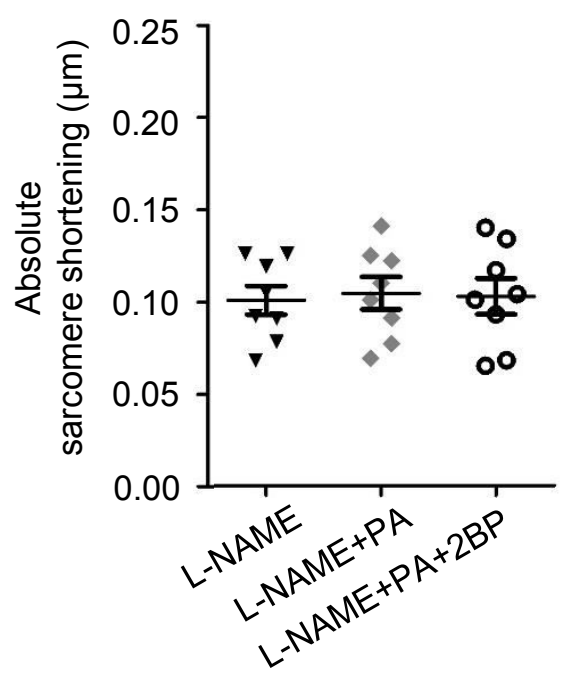


A

HTN
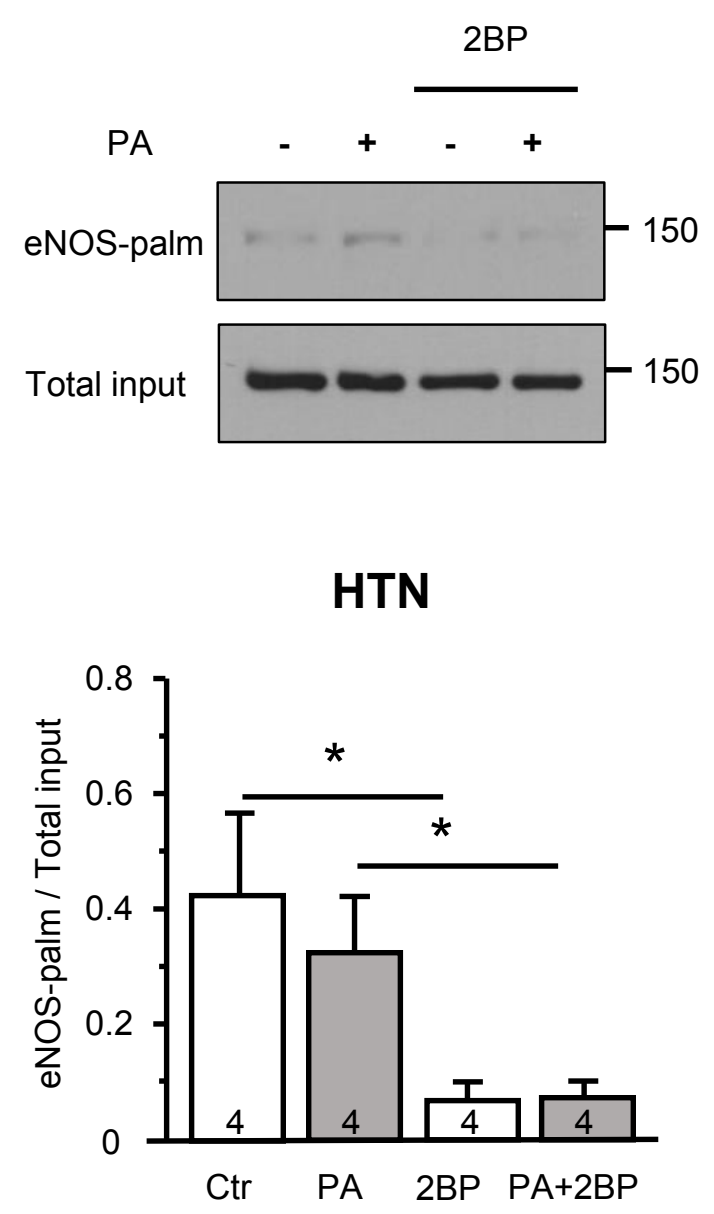

B

HTN

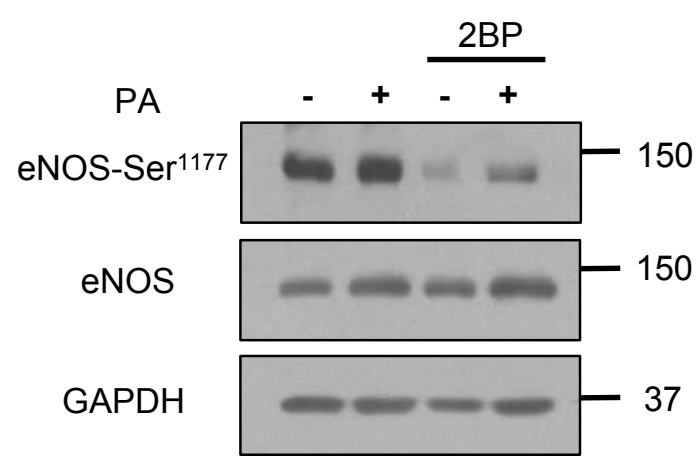

HTN

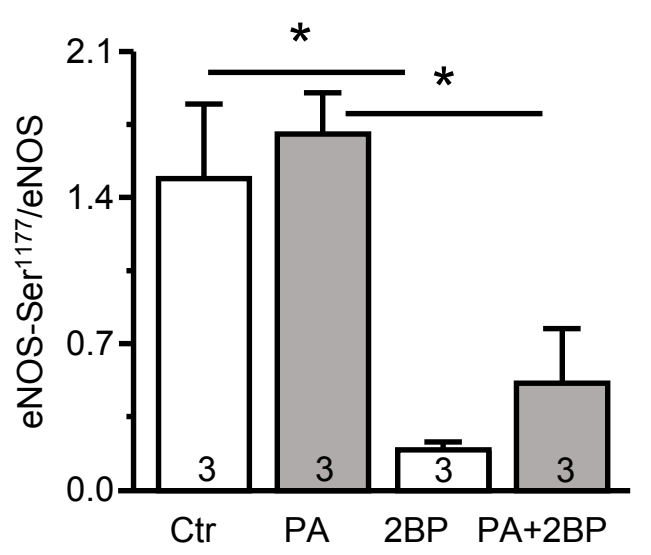

C

HTN

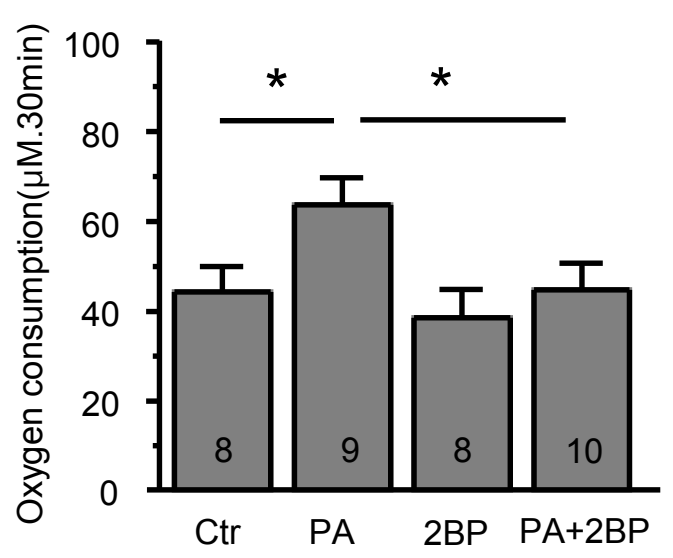



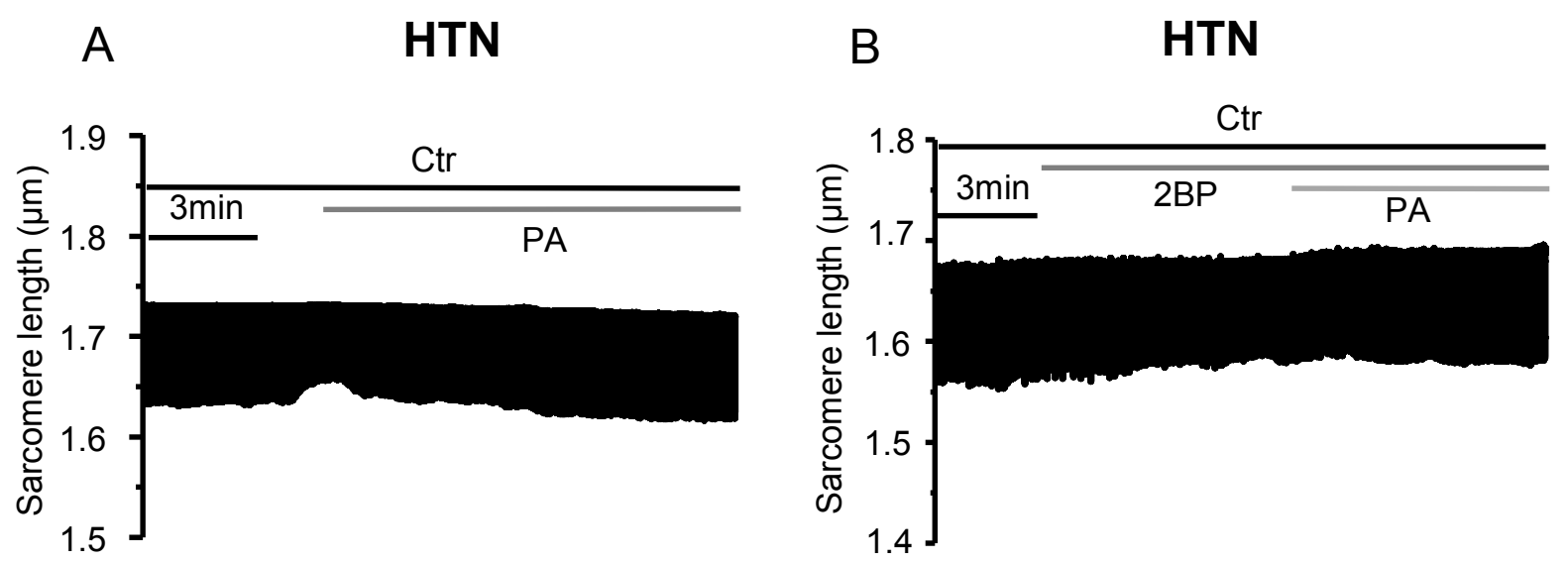

C HTN

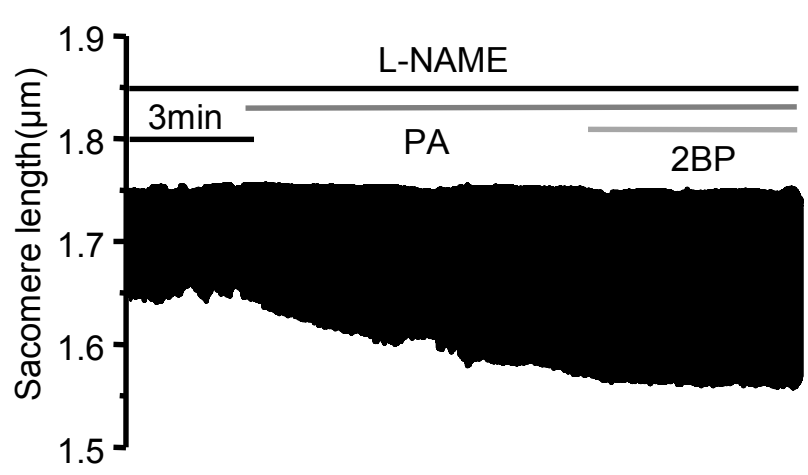

D

HTN

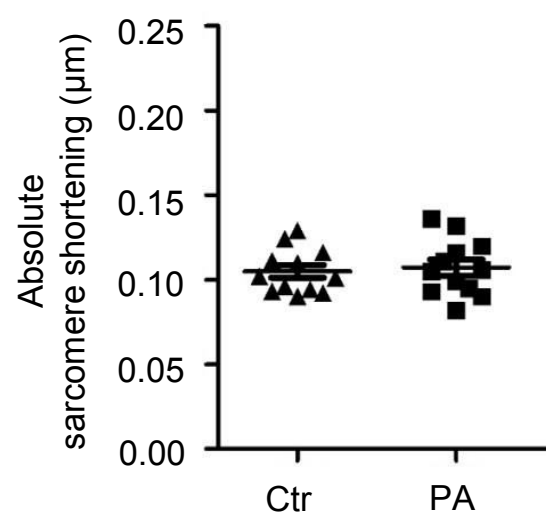

E

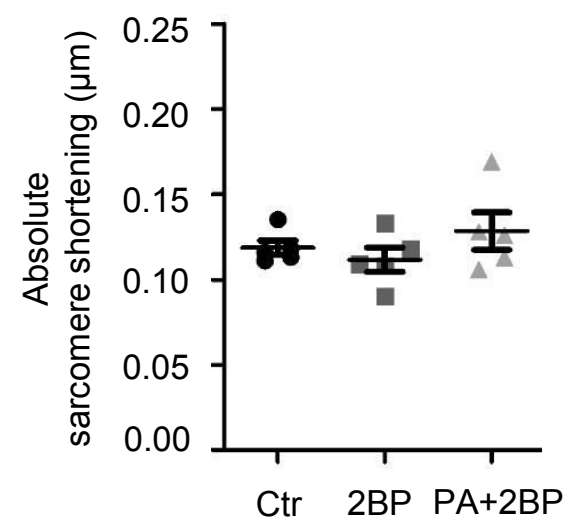

F

HTN

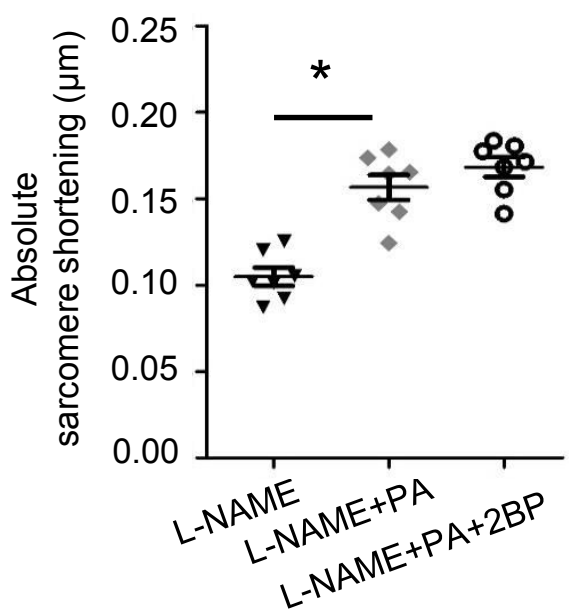

